\title{
CANCELLATION OF GROUPS WITH MAXIMAL CONDITION
}

\author{
R. HIRSHON
}

ABSTRACT. It is not true that a group which obeys the maximal condition for normal subgroups may always be cancelled in direct products. However, we show the following

THEOREM. Let $C$ be a group which obeys the maximal condition for normal subgroup. Suppose further that if $C_{*}$ is an arbitrary homomorphic image of $C$, then $C_{*}$ is not isomorphic to a proper normal subgroup of itself. Then $C$ may be cancelled in direct products.

Some generalizations of this result are indicated.

Let $A \times B$ represent the direct products of the groups $A$ and $B$. We shall say that $B$ may be cancelled in direct products if

$$
A \times B=A_{1} \times B_{1}, \quad B \approx B_{1}
$$

imply $A \approx A_{1}$ for any $A$. It seems natural to inquire about those groups which may be cancelled in direct products. The fact that a finite group may be cancelled appears to have been discovered as recently as 1947 [4, introduction]. From the work of Crawley and Jónsson, [1], one may show that a group obeying the minimal condition for normal subgroups may be cancelled. For if the center of a group has the exchange property, then so does the group. Furthermore, any abelian group with the minimal condition has the exchange property so that any group with the minimal condition has the exchange property. But a group with the minimal condition for normal subgroups is a direct product of finitely many indecomposable groups. Since any indecomposable group having the exchange property may be cancelled, a group which obeys the minimal condition for normal subgroups may be cancelled. ${ }^{1}$

The situation with groups which obey the maximal condition for normal subgroups is quite different. In [2], it is shown that an infinite cyclic group may not be cancelled in direct products. In this paper we will prove the following

THEOREM 1. Let $C$ be a group which obeys the maximal condition for normal subgroups. Suppose further that if $C_{*}$ is an arbitrary homomorphic image of $C$ then $C_{*}$ is not isomorphic to a proper normal subgroup of itself. Then $C$ may be cancelled in direct products.

Received by the editors May 16, 1969.

1 The author expresses his appreciation to Professor Peter Crawley for pointing this out to him. 
Proof. Deny. Say $A \times C=\bar{A} \times \bar{C}, C \approx \bar{C}$ but $A$ and $\bar{A}$ not isomorphic. Consider the pairs of groups $(L, C / H)$ such that there exists a corresponding pair of groups $\left(L_{1}, C_{1}\right)$ such that $L \times C / H=L_{1} \times C_{1}$, and such that $C / H$ and $C_{1}$ are isomorphic but $L$ and $L_{1}$ are not isomorphic. For example one such pair is $L=A, C / H=C$. Among these pairs there is a pair $(D, B)$ with $B=C / N$ such that $N$ is maximal. Let

$$
G=D \times B=D_{1} \times B_{1}
$$

where $B$ and $B_{1}$ are isomorphic but $D$ and $D_{1}$ are not. Now we note that $B \cap D_{1} \neq 1$, and $B_{1} \cap D \neq 1$. For if say $B \cap D_{1}=1, B \approx\left(B \times D_{1}\right) / D_{1}$ $\approx$ a normal subgroup of $G / D_{1} \approx B_{1}$. Our hypothesis concerning the homomorphic images of $C$ would then guarantee $B \times D_{1}=G$ so that $D \approx G / B \approx D_{1}$ contrary to hypothesis. Similarly, $B_{1} \cap D \neq 1$. Set $F$ $=B \cap D_{1} \neq 1, K=B_{1} \cap D \neq 1$. Now from (1), we may see

$$
G /(F \times K)=(B \times D) /(F \times K)=\left(B_{1} \times D_{1}\right) /(K \times F) .
$$

By a standard isomorphism theorem, we see from (2) $(B / F) \times(D / K)$ $\approx\left(B_{1} / K\right) \times\left(D_{1} / F\right)$. Hence, since $B \approx B_{1}$, we may write

$$
B \times(B / F) \times(D / K) \approx B_{1} \times\left(B_{1} / K\right) \times\left(D_{1} / F\right) .
$$

However,

$$
\begin{aligned}
B \times(B / F) \times(D / K) & \approx[B \times(D / K)] \times B / F \\
& \approx[(B \times D) / K] \times B / F \\
& =\left[\left(B_{1} \times D_{1}\right) / K\right] \times B / F \\
& \approx\left(B_{1} / K\right) \times D_{1} \times B / F
\end{aligned}
$$

In summary, we have

$$
B \times(B / F) \times D / K \approx\left(B_{1} / K\right) \times D_{1} \times B / F .
$$

Note that our hypothesis is symmetrical in $B$ and $B_{1}$ and $D$ and $D_{1}$, so if we interchange $B$ and $B_{1}$ and $D$ and $D_{1}$ (and hence $F$ and $K$ ), we see from (4)

$$
B_{1} \times\left(B_{1} / K\right) \times D_{1} / F \approx(B / F) \times D \times B_{1} / K .
$$

Now note from (3) that the groups on the left-hand sides of (4) and (5) are isomorphic. Consequently, the groups on the right-hand sides of (4) and (5) are isomorphic; that is,

$$
L_{1}=D_{1} \times(B / F) \times\left(B_{1} / K\right) \approx D \times(B / F) \times\left(B_{1} / K\right)=L_{2} .
$$

Hence by using an isomorphism of $L_{1}$ onto $L_{2}$ we may write (6) as an equality in the form,

$$
D \times B / F \times B_{1} / K=D_{2} \times E \times E_{1}
$$


where $D_{2} \approx D_{1}, E \approx B / F$ and $E_{1} \approx B_{1} / K$. Now set $M=D \times B / F$ and $M_{1}=D_{2} \times E$. Then $M$ and $M_{1}$ are not isomorphic or we arrive at a contradiction of the maximality of $N$. Hence (7) may be written as

$$
M \times B_{1} / K=M_{1} \times E_{1}, \quad M \neq M_{1}
$$

which again gives rise to a contradiction of the maximality of $N$ and so our result is proven.

We note that we can state a slightly stronger result than Theorem 1. We have

Theorem 1*. Suppose $C$ obeys the maximal condition for normal subgroups. Suppose further that if $C_{*}$ is an arbitrary homomorphic image of $C$ and if $C_{i}$ is a descending series of normal subgroups of $C_{*}$, $C_{i+1} \subset C_{i}$ with each $C_{i}$ isomorphic to $C_{*}$, then ultimately the $C_{\boldsymbol{i}}$ are identical. Then $C$ may be cancelled in direct products.

Proof. The proof is exactly the same as the proof of Theorem 1 except that one notices that if

$$
G=A \times B=A_{1} \times B_{1}, \quad B \approx B_{1}
$$

and if $A \cap B_{1}=1$, but $A \times B_{1}$ is a proper subgroup of $G$, then $B$ has a descending, nonterminating series of normal subgroups, each isomorphic to $B$. For $A \times B_{1}=A \times \bar{B}, \bar{B}$ a proper normal subgroup of $B$. Let $\alpha$ be the projection of $G$ onto $B$; that is

$$
(a b) \alpha=b \quad \text { for } a \in A, \quad b \in B .
$$

Let $\theta$ be an isomorphism of $B$ onto $B_{1}$. Then if $\gamma=\theta \alpha$, then $\gamma$ is an isomorphism of $B$ onto $\bar{B}$ and the groups $B \gamma^{j}, j \geqq 0$, form a nonterminating descending series of isomorphic subgroups of $B$ and $B \gamma^{j}$ is a normal subgroup of $B$, since both $\theta$ and $\alpha$ map normal subgroups into normal subgroups.

We observe that we could have stated Theorem $1 *$ more generally for operator groups.

Some applications of this cancellation result appear in [3].

\section{REFERENCES}

1. P. Crawley and B. Jónsson, Refinements for infinite direct decompositions of algebraic systems, Pacific J. Math. 14 (1964), 797-855. MR 30 \#49.

2. R. Hirshon, On cancellation in groups, Amer. Math. Monthly (to appear).

3. - Some theorems on hopficity, Trans. Amer. Math. Soc. 141 (1969), 229244.

4. B. Jónsson and A. Tarski, Direct decompositions of finite algebraic systems, Notre Dame Mathematical Lectures, no. 5, University of Notre Dame, Notre Dame, Ind., 1947. MR 8, 560.

Polytechnic Institute of BRooklyn 